\title{
Neoplastic progression of the human breast cancer cell line G3S1 is associated with elevation of cytoskeletal dynamics and upregulation of MT1-MMP
}

\author{
ONDREJ TOLDE ${ }^{1}$, DANIEL RÖSEL ${ }^{1}$, CLAUDIA T. MIERKE ${ }^{3}$, DANIELA PANKOVÁ ${ }^{1}$, \\ PETR FOLK $^{1}$, PAVEL VESELY ${ }^{2}$ and JAN BRÁBEK ${ }^{1}$ \\ ${ }^{1}$ Department of Cell Biology, Faculty of Science, Charles University in Prague; ${ }^{2}$ Institute of Molecular Genetics AS, \\ Prague, Czech Republic; ${ }^{3}$ Biophysics Group, Department of Physics, University of Erlangen-Nuremberg, Germany
}

Received November 25, 2009; Accepted January 16, 2010

DOI: 10.3892/ijo_00000560

\begin{abstract}
The newly established breast cancer cell line G3S1, derived from EM-G3 breast cancer progenitors, was analyzed for functional changes related to neoplastic progression manifested by elevated invasiveness and enhanced capability to degrade gelatin. Degradation of gelatin and invasiveness of G3S1 cells was found to be dependent on the activity of matrix proteinases and actin cytoskeletal dynamics. Therefore, the expression and activity of these proteases was compared in G3S1 and EM-G3 cells. Despite enhanced capability of G3S1 cells to degrade gelatin, these cells exhibited lower levels of secreted extracellular matrix degrading proteases than parental EM-G3 cells. However, the expression of membrane-bound MT1-MMP was strongly elevated in G3S1 cells. While the degradation of gelatin was associated with invadopodia-like structures in both EM-G3 and G3S1 cells, the cytoskeletal remodeling dynamics was greatly elevated in G3S1 cells, suggesting that upregulation of MT1MMP, together with elevation of cytoskeletal remodeling dynamics can effectively cause elevated invasiveness and enhanced matrix degrading capability in G3S1 cells.
\end{abstract}

\section{Introduction}

Neoplastic progression in carcinoma cells is often associated with an increased capacity for cell invasion through barriers of extracellular matrix (1). Two main modes of overcoming the barrier of the surrounding tissue by tumor cells were described: the proteolysis dependent mode requiring extracellular proteolysis and the recently described proteolysis independent amoeboid mode depending on the activity of the

Correspondence to: Dr Jan Brábek, Department of Cell Biology, Faculty of Science, Charles University in Prague, Vinicna 7, 12843 Prague, Czech Republic

E-mail: brabek@natur.cuni.cz

Key words: invasiveness, breast carcinoma, neoplastic progression, MT1-MMP, cytoskeletal dynamics
Rho kinase ROCK $(2,3)$. In amoeboid mode, the upregulation of ROCK was shown to result in the generation of sufficient actomyosin force to deform collagen fibers and give tumor cells ability to push through the extracellular matrix (ECM) $(4,5)$. In contrast, protease dependent cell motility is associated with elevated expression and activation of extracellular matrix degrading proteases and substantial changes in structure and dynamics of actin cytoskeleton (6). Extracellular proteases remodel the ECM to drive the dissemination of cancer cells into normal adjacent tissue. The proteases implicated in the process of tumor invasion and metastasis include, among others, metalloproteases, serine proteases and cathepsins (reviewed in ref. 7). In general, cancer cells have elevated levels of proteases belonging to more than one class (7).

In addition to the activation of extracellular proteases, changes in actin cytoskeletal structure and dynamics were found to be very important for protease-dependent mode of invasion. The major actin-rich adhesive structures, responsible for cellular invasion, are podosomes and invadopodia. Both structures establish close contact to the substrate but can also degrade components of the ECM. Focal degradation of extracellular matrix localized at podosomes or invadopodia is thought to contribute to cellular invasiveness in both physiological and pathological situations. While podosomes are formed in cell types such as monocytes, endothelial and smooth muscle cells, invadopodia, structurally close related proteolytically active plasma-membrane protrusions, were found in carcinoma cells and Src-transformed fibroblasts (reviewed in refs. 8-10). Podosomes and invadopodia are very important, but probably not solely responsible for invasion related alterations in actin structure and dynamics. Other structures and mechanisms were also suggested to take part in the local modulation of cytoskeletal structures such as contractile forces, the turnover of substrate adhesions and their associated microfilaments that were required for cell invasion $(11,12)$.

Previously, we established a new breast cancer cell line G3S1, derived from EM-G3 breast cancer progenitors by chronic nutritional stress and treatments with tumor promoter TPA (12-O-tetradecanoylphorbol-13-acetate). G3S1 cells exhibited elevated invasiveness in Matrigel invasion chambers as compared to parental EM-G3 cells. Elevated invasiveness 
of G3S1 cells was accompanied by higher incidence of specific dynamic morphotypes myzitiras (sucker-like) and newly observed vthela morphotype (leech-like) (13).

In this study, we analyzed functional changes associated with neoplastic progression of G3S1 cells emphasizing expression and activation of extracellular proteases as well as actin cytoskeletal structures and dynamics.

\section{Materials and methods}

Cell culture. EM-G3 cells were maintained in H-MEM (Invitrogen, Carlsbad, CA) supplemented with non-essential amino acids, $0.5 \mu \mathrm{g} / \mathrm{ml}$ hydrocortisone, $5 \mu \mathrm{g} / \mathrm{ml}$ insulin, $10^{-10} \mathrm{M}$ cholera toxin, $5 \mathrm{ng} / \mathrm{ml}$ epidermal growth factor (EGF), $10 \%$ bovine and $2 \%$ fetal bovine serum, penicillin, and streptomycin (EMA medium, all supplements from Sigma, St. Louis, MO) while G3S1 cells also grew in H-MEM supplemented with non-essential amino acids with $10 \%$ bovine serum only.

Invasion assays. Invasion assay was performed according to the manufacturer's protocol (Oris ${ }^{\mathrm{TM}}$ Cell Invasion \& Detection Assay, Platypus Technologies, Madison, WI). Briefly, 4x10 cells were seeded in individual wells of basement membrane extract (BME) coated 96-well plate with a stopper to restrict cell seeding to the outer annular regions of the wells. Removal of the stopper reveals a round unseeded region, into which the seeded cells invade. Cells were then overlaid with BME to create 3 -dimensional invasion matrix. Invasion was assessed after 3 days using Nikon-Eclipse TE2000-S microscope as a decrease of cell-free area quantified by NIS-Elements software.

Immunoblotting. Subconfluent cell cultures were washed with phosphate buffered saline (PBS) and lysed in modified RIPA buffer [50 mM Tris- $\mathrm{HCl}$ (pH 7.4), $150 \mathrm{mM} \mathrm{NaCl}, 5 \mathrm{mM}$ EDTA, 1\% NP40, 1\% sodium deoxycholate, $50 \mathrm{mM} \mathrm{NaF}$, $1 \%$ aprotinin and $\left.0.1 \mathrm{mM} \mathrm{Na} \mathrm{VO}_{4}\right]$. Protein concentrations in lysates were determined using the BCA assay (Pierce). Lysates equivalent to $10-30 \mathrm{mg}$ protein were diluted in $2 \mathrm{X}$ SDS-PAGE sample buffer for immunoblot analysis of wholecell extracts. For immunoblotting, samples were separated on $10 \%$ SDS-polyacrylamide gels and transferred onto nitrocellulose membranes. Non-specific activity was blocked by incubating $45 \mathrm{~min}$ at room temperature in Tris-buffered saline containing $0.05 \%$ Tween-20 (TBST) and $4 \%$ bovine serum albumin (BSA). Membranes were then incubated overnight in primary antibody, washed extensively with TBST, and then incubated for $1 \mathrm{~h}$ at room temperature with HRP-conjugated secondary antibody. After extensive washing in TBST, the blots were developed by enhanced chemiluminescence and exposed to autoradiographic film. Primary antibodies against MT1-MMP, uPA and LIMK1 were purchased from Santa Cruz Biotechnology Inc. (Santa Cruz, CA), phospho-cofilin antibody was from Cell Signaling Technology (Danvers, MA). Secondary HRP-conjugated antibodies were from Santa Cruz Biotechnology Inc.

In gel gelatin zymography. In total, $2 \times 10^{5}$ cells were plated per well of a 24 well plate. After $16 \mathrm{~h}$, cells were washed with PBS and incubated in $300 \mu \mathrm{l}$ of serum-free media for $48 \mathrm{~h}$. Aliquots $(25 \mu \mathrm{l})$ of the conditioned media were loaded for zymography on a $10 \%$ SDS-PAGE gel containing $1 \mathrm{mg} / \mathrm{ml}$ gelatin as described (36). Briefly, gel proteins were washed for $1 \mathrm{~h}$ in $50 \mathrm{mM}$ Tris- $\mathrm{HCl}(\mathrm{pH} 7.5), 0.1 \mathrm{M} \mathrm{NaCl}, 2.5 \%$ Triton $\mathrm{X}-100$, and then incubated at $37^{\circ} \mathrm{C}$ in $50 \mathrm{mM}$ Tris- $\mathrm{HCl}$ (pH 7.5), $10 \mathrm{mM} \mathrm{CaCl}$ and $0.02 \% \mathrm{NaAzide}$ for $17 \mathrm{~h}$. The gels were stained with Coomassie Brilliant Blue and destained in $7 \%$ acetic acid/5\% methanol.

FITC-gelatin zymography. Coverslips were coated with FITCgelatin $(1 \mathrm{mg} / \mathrm{ml})$, air dried, rehydrated with water for $15 \mathrm{~min}$ in $4^{\circ} \mathrm{C}$ and fixed with $0.5 \%$ glutaraldehyde for $30 \mathrm{~min}$ in $4^{\circ} \mathrm{C}$. Cells were grown on FITC-gelatin coated coverslips for $48 \mathrm{~h}$ and then fixed in $4 \%$ paraformaldehyde (30 min), permeabilized in $0.5 \%$ Triton $\mathrm{X}-100$, washed three times with PBS, blocked in 3\% BSA and then stained with Alexa 594 phalloidin (Molecular Probes, Eugene, OR). Coveslips were then washed four times and mounted. Images were acquired by Leica TCS SP2 microscope system using Leica $63 \mathrm{x} / 1.45$ oil objective. Concentrations of inhibitors used in FITC-gelatin zymography were: GM6001 $10 \mu \mathrm{M}$ (Sigma), aprotinin $100 \mu \mathrm{g} / \mathrm{ml}$ (Serva, Heidelberg, Germany), E-64 $50 \mathrm{nM}$ (Serva), latrunculin A $100 \mathrm{nM}$ (Sigma).

Cytoskeletal remodeling dynamics (nanoscale particle tracking). Cells $(200,000)$ of $80 \%$ confluent EM-G3 or G3S1 cells were seeded into $\varnothing 3.5 \mathrm{~cm}$ dishes. After overnight culture, fibronectin $(\mathrm{FN})$ coated beads were bound to the cells for $30 \mathrm{~min}$ at $37^{\circ} \mathrm{C}, 5 \% \mathrm{CO}_{2}$ and $95 \%$ humidity. The position of beads was tracked for 5 min from bright-field images recorded with a CCD camera (ORCA ER) at a frame rate of $8.3 \mathrm{~Hz}$. An intensity-weighted center of mass algorithm was used to determine the bead positions with $10 \mathrm{~nm}$ accuracy (14). These beads moved spontaneously with a mean square displacement (MSD) that also followed a power-law with time, $\mathrm{MSD}=\mathrm{D} \cdot\left(\mathrm{t} / \mathrm{t}_{0}\right)^{\beta}+\mathrm{c}$. An apparent diffusivity $\mathrm{D}^{*}$ was calculated as the squared distance a bead had traveled during a $1 \mathrm{sec}$ time interval, averaged over all recording times and beads $(16,38)$. The persistence of motion can be described, by the power-law exponent, $\beta$. c reflects random noise from thermal and non-thermal sources such as single myosin motors, and $\mathrm{t}_{0}$ was arbitrarily set to $1 \mathrm{sec}$. The fit parameters were determined by a least-squares fit $(15,16)$.

\section{Results}

G3S1 cells have greater capability to degrade gelatin than EM-G3 progenitors. The neoplastic progression has often been found to be associated with increased capability of the cells to degrade ECM (17). We employed FITC-gelatin zymography to examine whether neoplastic progression of G3S 1 cells is accompanied with increased capability to degrade ECM compounds. We found that the degradation of FITC-gelatin was substantially increased in G3S1 cells when compared to parental EM-G3 cells (Fig. 1A).

In carcinomas, the degradation of ECM is associated with specific actin-rich cellular structures called invadopodia (10). We analyzed the sites of FITC-gelatin degradation using a confocal laser scanning microscopy and found that both EM-G3 and G3S1 cells form invadopodia-like structures, seen as protruding F-actin rich structures colocalizing with sites of FITC-gelatin degradation (Fig. 1B). 
$\mathbf{a}$

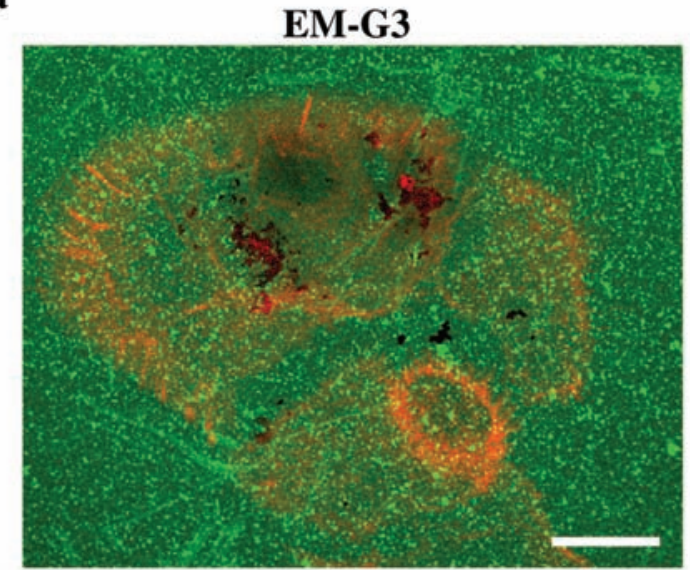

b

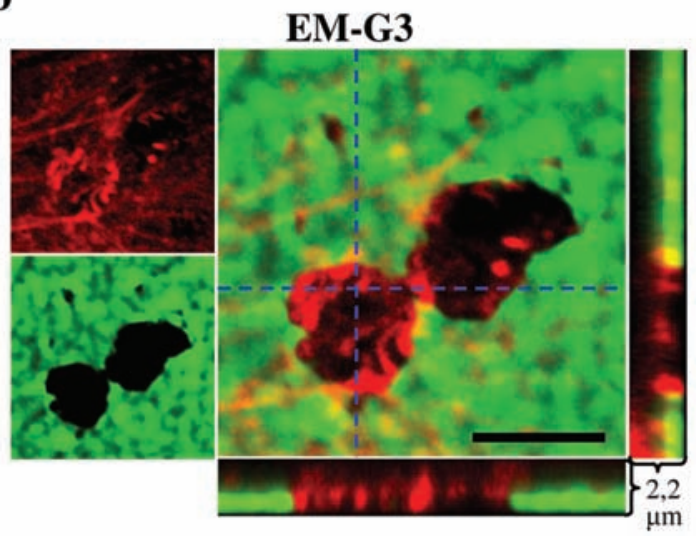

G3S1

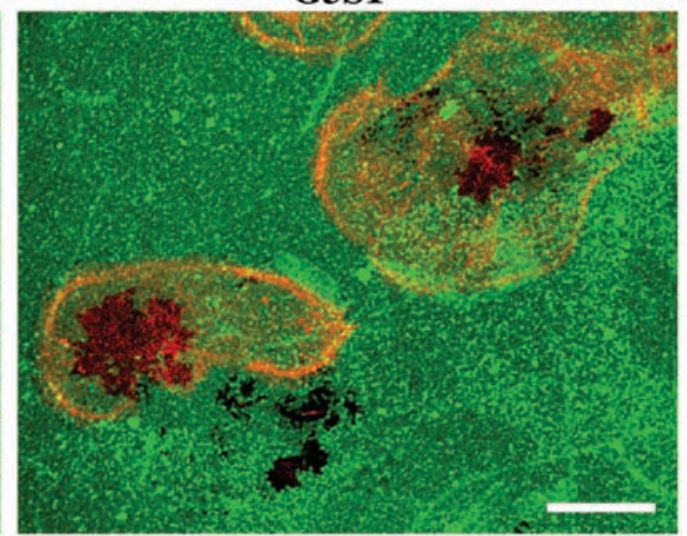

G3S1

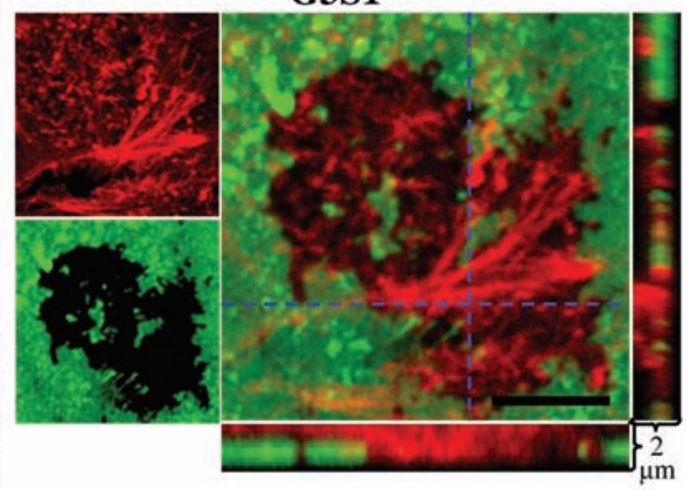

Figure 1. G3S1 cells degrade gelatin more effectively than EM-G3 cells. (a) EM-G3 and G3S1 cells were grown on FITC-gelatin coated cover slips and stained for F-actin. (b) Detailed view of sites of degradation. Only inner sections of ventral sides of cells are shown. Bottom and right images show cross sections (positions indicated by dashed line, oriented so that outer edge of the image represents the bottom), where protrusions of actin structures are apparent. Scale bars: $20 \mu \mathrm{m}$ (A), $5 \mu \mathrm{m}$ (B).

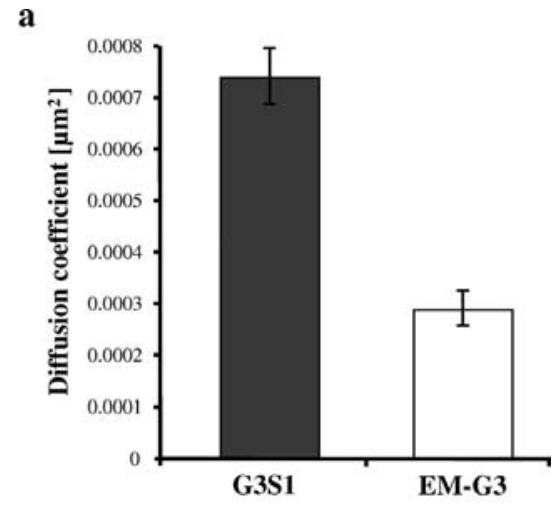

b

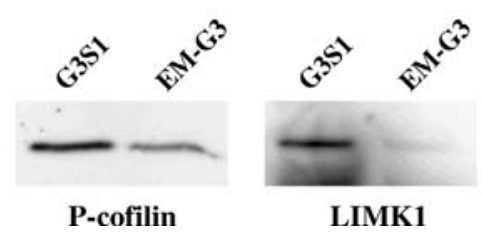

Figure 2. Increased cytoskeletal remodeling dynamics in G3S1 cells. (a) We used nanoscale particle tracking of FN-coated beads that were bound to the actin cytoskeleton via integrins. The apparent diffusivity, D (describing the speed of cytoskeletal remodeling processes), was 2-fold increased in G3S1 cells compared to EM-G3 cells (bars are mean \pm SE). (b) Western blot analyses show that G3S1 cells expressed higher levels of phosphorylated cofilin and more LIMK1 compared to EM-G3 cells. The results are representative of at least two independent experiments.
Elevated invasiveness of G3S1 cells is accompanied by increased cytoskeletal remodeling dynamics. Alterations in actin cytoskeletal structures and dynamics were generally found to be important for protease-dependent mode of invasion (reviewed in ref. 18). Therefore, we analyzed whether differences in invasiveness of G3S1 and EM-G3 cell lines correlate with alterations in cytoskeletal remodeling dynamics. To measure the cytoskeletal dynamics in EM-G3 and G3S1 cells, we employed nanoscale particle tracking method, because of high accuracy and possibility of direct visualization of particles movement representing ATPdriven cytoskeletal rearrangements. Nanoscale particle tracking analyzes the random walk of unforced, spontaneously diffusing beads. In this study, $4.5 \mu \mathrm{m}$ diameter carboxylated polystyrene beads were coated with human fibronectin and attached to subconfluent cells. The beads bind to integrins at the cell surface, initiate focal contact formation, and anchor firmly to the actin cytoskeleton. The beads cannot move unless the focal contacts and actin structures to which they are attached rearrange (19). ATP-driven cytoskeletal rearrangements can be quantified by an apparent diffusivity $\mathrm{D}^{*}$ of the beads, measured as the squared distance of bead motion during a $1 \mathrm{sec}$ time interval. The diffusion coefficient $\mathrm{D}^{*}$ was significantly increased in G3S1 cells compared to EM-G3 cells (Fig. 2A), suggesting that $\mathrm{G} 3 \mathrm{~S} 1$ cells exhibit higher cytoskeletal dynamics when compared to EM-G3 cell. 
a

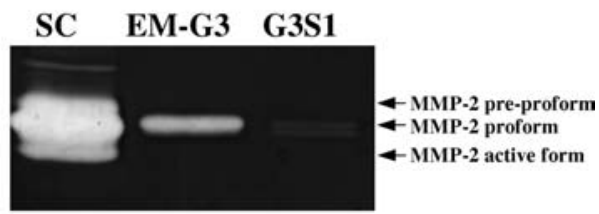

b

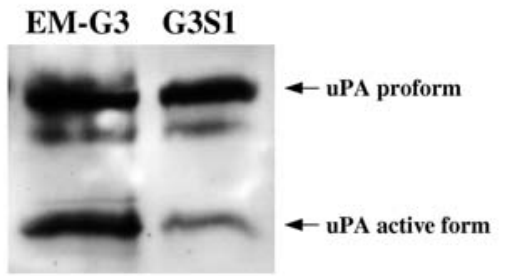

c

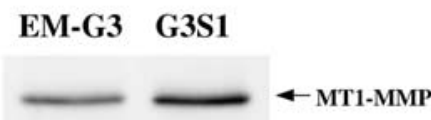

Figure 3. Expression of matrix-degrading proteases in EM-G3 and G3S1 cells. EM-G3, G3S1 and control SC cells were grown in 24-wells plates in serumfree medium. After $48 \mathrm{~h}$, the medium was collected and equal amounts were subjected to (a) gelatin zymography, (b) casein zymography or (c) immunoblotting. The results are representative of at least two independent experiments.
The higher cytoskeletal remodeling dynamics is often associated with increase phosphorylation of cofilin, an actin severing protein (20). We have found increased cofilin phosphorylation and increased expression of cofilin regulating kinase LIMK1 in G3S1 cells (Fig. 2B), which further supports the observation of elevated actin cytoskeletal remodeling dynamics in G3S1 cells.

Membrane-bound MT1-MMP but not secreted proteases are elevated in G3S1 cells. Degradation of extracellular matrix compounds by breast cancer cells is often associated with elevated expression and/or activation of matrix proteinases (reviewed in ref. 21). To examine which proteinases are responsible for increased ECM degradation, we performed zymography assays on the conditioned media of G3S1 and EM-G3 cells. We detected as the major gelatinase activity in the region of the zymograms corresponding to MMP-2 (gelatinase A). Surprisingly, the less invasive EM-G3 cells had greatly elevated secretion of MMP-2 (Fig. 3A). As the breast cancer progression is often accompanied with increased secretion of urokinase plasminogen activator uPA (22), we next examined the expression of uPA and again, unexpectedly, we found that less invasive EM-G3 cells secrete more uPA (Fig. 3B).

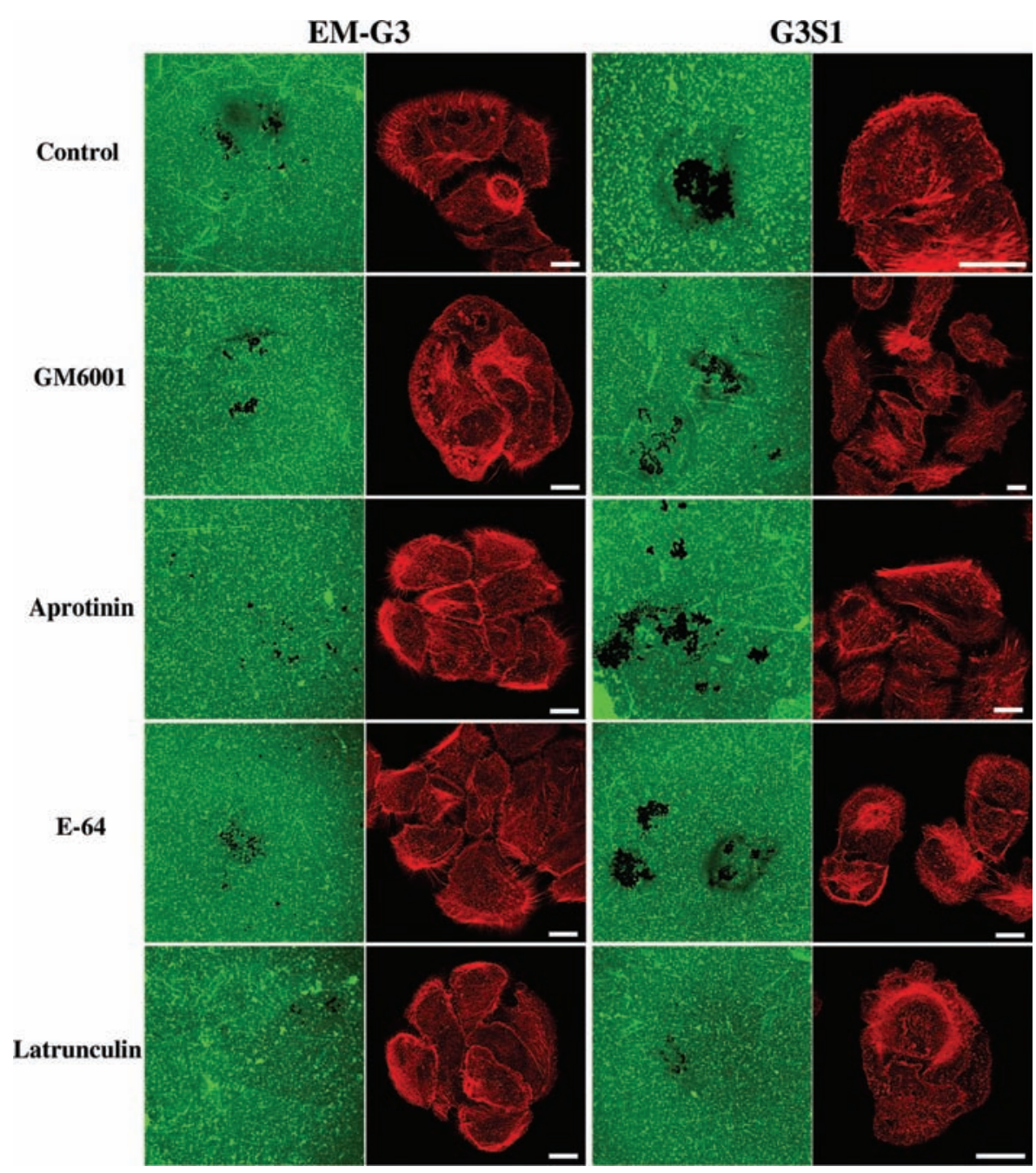

Figure 4. Sensitivity of gelatin degradation to selected inhibitors in EM-G3 and G3S1 cells. EM-G3 (left pannel) and G3S1 (right pannel) cells were treated with $10 \mu \mathrm{M}$ GM6001, $100 \mu \mathrm{g} / \mathrm{ml}$ aprotinin, $50 \mathrm{nM} \mathrm{E-64}$ and $100 \mathrm{nM}$ latrunculin. Scale bars: $10 \mu \mathrm{m}$. 
a

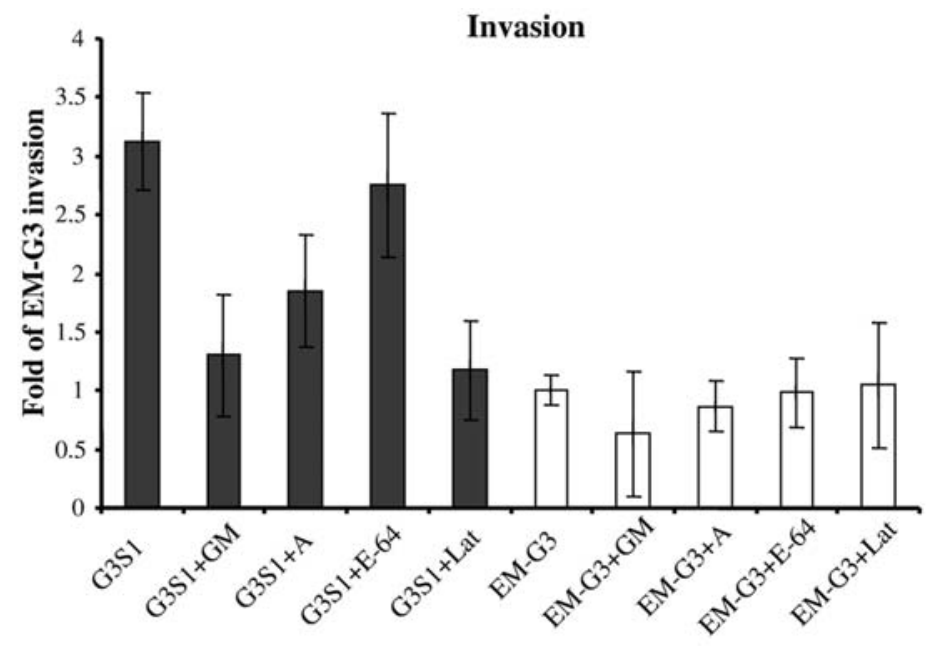

b

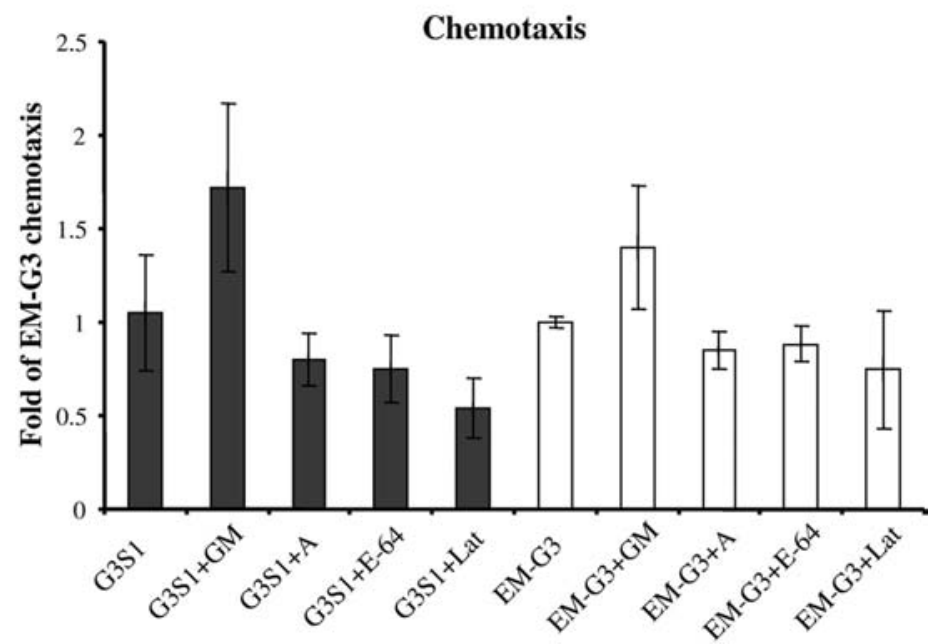

Figure 5. Invasiveness of G3S1 cells is MMP-dependent. Cell invasion (a) and chemotaxis (b) assay in presence of $20 \mu \mathrm{M} \mathrm{GM6001} \mathrm{(+GM),} 100 \mu \mathrm{g} / \mathrm{ml}$ aprotinin (+A), $50 \mathrm{nM} \mathrm{E-64} \mathrm{(+E)} \mathrm{and} 100 \mathrm{nM}$ latrunculin (+Lat). Cell invasion was calculated as \% of not treated EM-G3 cells and averaged from at least three independent experiments performed in triplicates. The data are presented as mean $\pm \mathrm{SD}$.

Results from recent in vitro studies highlight that a membrane-bound MT1-MMP but not secreted MMPs, is critical for both the breaching of basement membranes by tumor cells and cell invasion through interstitial type I collagen tissues (23). Therefore, we next analyzed the expression of membrane-bound matrix metalloproteinase MT1-MMP and found it greatly increased in G3S1 cells (Fig. 3C), suggesting that MT1-MMP could be responsible for elevated gelatin degradation by $\mathrm{G} 3 \mathrm{~S} 1$ cells.

Gelatin degradation capability of EM-G3 and G3S1 cells is sensitive to protease inhibitors and latrunculin. To further confirm the role of matrix metalloproteinases and cytoskeletal dynamics in elevated gelatin degradation by G3S1 cell line, we performed gelatin degradation assays with general class specific protease inhibitors and actin polymerization inhibitor latrunculin. FITC-gelatin zymography assays were performed with general metalloproteinase inhibitor GM6001, uPA proteinase inhibitor aprotinin, cysteine cathepsin inhibitor E-64 and latrunculin. In the presence of GM6001 the ability of both G3S1 and EM-G3 cells to degrade FITC-gelatin was greatly decreased (Fig. 4), suggesting that matrix metallopro- teinases are mostly responsible for the degradation of gelatin by G3S1 and EM-G3 cells. Aprotinin was also able to decrease to some extent FITC-gelatin degradation by EM-G3 and G3S1 cells, suggesting the possible role of uPa proteinase in gelatin degradation by EM-G3 and G3S1 cells. In contrast, cysteine cathepsin inhibitor E64 did not significantly influence the degradation of FITC-gelatin by either EM-G3 or G3S1 cells. Surprisingly, the most inhibitory for gelatin degradation was treatment with very low dose of latrunculin $(100 \mathrm{nM})$. At $100 \mathrm{nM}$ concentration latrunculin had only negligible effect on cell morphology (Fig. 4), and only moderate effect on chemotaxis (Fig. 5B), suggesting that the effects of $100 \mathrm{nM}$ latrunculin reflect more probably a decrease of actin cytoskeletal dynamics than disruption of actin cytoskeleton integrity.

Increased invasiveness of G3S1 is MMP-dependent and sensitive to latrunculin. To determine relative contribution of matrix proteases and cytoskeletal dynamics on invasiveness of EM-G3 and G3S1 cells, we performed 3D invasion assays with class-specific inhibitors of selected extracellular proteinases and with actin polymerization inhibitor latrunculin. 
Indeed, we found that the invasiveness of both cell lines was greatly inhibited by general MMP inhibitor GM6001 (Fig. 5A) in spite of increased chemotaxis in the presence of this inhibitor (Fig. 5B). Inhibition of MMPs in G3S1 cells using $10 \mu \mathrm{M}$ GM6001 resulted in a $60 \%$ decrease in invasion, lowering the invasion efficiency close to the invasion efficiency of EM-G3 cells. uPA proteinase inhibitor aprotinin also lowered the invasion rate to some extent (ca $41 \%$ decrease in G3S1 cells); however, this could also be at least partly attributed to the decrease in chemotaxis (ca 24\% in G3S1 cells) in the presence of this inhibitor. Cysteine cathepsins inhibitor E-64 did not significantly influenced the invasiveness of either EM-G3 or G3S1 cells. Consistent with the profound effect of latrunculin on gelatin degradation by G3S1 cells, the treatment with $100 \mathrm{nM}$ latrunculin resulted in $63 \%$ decrease of the G3S1 invasiveness whereas the invasiveness of EM-G3 cells was not significantly altered (Fig. 5A). Notably the great decrease of invasiveness of G3S1 cells was achieved in spite of increase of active MMP-2 secretion in both EM-G3 and G3S1 cells after $100 \mathrm{nM}$ latrunculin treatment (data not shown). Taken together, our data suggest that neoplastic progression of human breast cancer cell line G3S1 is associated with an MT1-MMP dependent increase of ECM degradation and an increase of actin cytoskeletal dynamics.

\section{Discussion}

Cancer cell invasion through surrounding tissue is a critical step in the process of metastatic transformation. In order to overcome the ECM barriers, tumor cells use different strategies; proteolysis- or force-dependent remodeling of the ECM $(24,25)$. Our data show that the invasive G3S1 cells can effectively degrade gelatin and that their invasion is inhibited by MMP and in part by uPA protease inhibitors, suggesting that the invasive G3S1 cells employ the proteolysis-dependent mode of invasion. Accumulated lines of evidence on MMPs in human cancer tissues have demonstrated that MT1-MMP plays an essential role in invasion and metastases in most cancers (reviewed in ref. 26). In this study we report an upregulation of MT1-MMP in more invasive G3S1 cells compared to EM-G3 cells. We hypothesize that the upregulation of MT1-MMP could be at least in part responsible for elevated MMP-dependent invasiveness of G3S1 cells.

Previous studies reported that at least four parameters such as adhesion strength, cytoskeletal remodeling dynamics, enzymatic matrix degradation and the generation and transmission of contractile forces regulated cell motility in a $3 \mathrm{D}$ ECM $(24,27)$. None of these parameters seems to be solely responsible for increased invasiveness, but the balance of all four determines the migratory behavior of cells. Our data show that besides the enzymatic degradation of the ECM, the cytoskeletal remodeling dynamics facilitates elevated cell invasion. We found that more invasive G3S1 cells exhibit greater cytoskeletal remodeling dynamics and in contrast to EM-G3 cells are sensitive to latrunculin treatment for invasion indicating that higher cytoskeletal remodeling dynamics of $\mathrm{G} 3 \mathrm{~S} 1$ cells is at least in part responsible for their increased invasiveness. This is also consistent with our recently published study reporting an increased cytoskeletal re- modeling of highly invasive tumor cells compared to weakly invasive (16).

Several invasive neoplasms, including head and neck squamous cell carcinoma, breast carcinoma, melanoma and glioma, contain tumor cells that can form actin-rich protrusions with ECM proteolytic activity called invadopodia. These dynamic organelle-like structures adhere to, and digest, collagens, laminins and fibronectin. The function of invadopodia is dependent on multiple transmembrane, cytoplasmic and secreted proteins engaged in cell adhesion, actin assembly, membrane regulation and ECM proteolysis (reviewed in ref. 28). While invadopodia were mostly observed in metastatic tumor cell lines, fewer reports describe the presence of invadopodia-like structures in primary tumor cells, e.g. primary mammary tumors (29), or primary head and neck squamous carcinoma cells (30). In this study, we document the presence of invadopodia-like structures in breast cancer progenitors $(31,13)$, further confirming the early onset of invadopodia-like structure formation during the process of malignant transformation.

\section{Acknowledgements}

We would like to thank Mrs. M. Charvátová and Mrs. C. Albert for excellent technical assistance. This study was supported by grants from the Czech Ministry of Education, Youth and Sport (Research Center grant LC06061) and (MSM0021620858) and the Deutsche Forschungsgemeinschaft (FA336/21) and Deutsche Krebshilfe (107384).

\section{References}

1. Terranova VP, Hujanen ES, Loeb DM, Martin GR, Thornburg L and Glushko V: Use of a reconstituted basement membrane to measure cell invasiveness and select for highly invasive tumor cells. Proc Natl Acad Sci USA 83: 465-469, 1986.

2. Sahai E and Marshall CJ: Differing modes of tumour cell invasion have distinct requirements for Rho/ROCK signalling and extracellular proteolysis. Nat Cell Biol 5: 711-719, 2003.

3. Wolf K, Mazo I, Leung H, Engelke K, von Andrian UH, Deryugina EI, Strongin AY, Brocker EB and Friedl P: Compensation mechanism in tumor cell migration: mesenchymalamoeboid transition after blocking of pericellular proteolysis. J Cell Biol 160: 267-277, 2003.

4. Wyckoff JB, Pinner SE, Gschmeissner S, Condeelis JS and Sahai E: ROCK- and myosin-dependent matrix deformation enables protease-independent tumor cell invasion in vivo. Curr Biol 16: 1515-1523, 2006.

5. Rosel D, Brabek J, Tolde O, Mierke CT, Zitterbart DP, Raupach C, Bicanova K, Kollmannsberger P, Pankova D, Vesely P, Folk $\mathrm{P}$ and Fabry B: Upregulation of Rho/ROCK signaling in sarcoma cells drives invasion and increased generation of protrusive forces. Mol Cancer Res 6: 1410-1420, 2008.

6. Friedl P: Prespecification and plasticity: shifting mechanisms of cell migration. Curr Opin Cell Biol 16: 1423, 2004.

7. Goel A and Chauhan SS: Role of proteases in tumor invasion and metastasis. Indian J Exp Biol 35: 553-564, 1997.

8. Linder S: The matrix corroded: podosomes and invadopodia in extracellular matrix degradation. Trends Cell Biol 17: 107-117, 2007.

9. Gimona M, Buccione R, Courtneidge SA and Linder S: Assembly and biological role of podosomes and invadopodia. Curr Opin Cell Biol 20: 235-241, 2008.

10. Buccione R, Caldieri G and Ayala I: Invadopodia: specialized tumor cell structures for the focal degradation of the extracellular matrix. Cancer Metastasis Rev 28: 137-149, 2009.

11. Spinardi L, Rietdorf J, Nitsch L, Bono M, Tacchetti C, Way M and Marchisio PC: A dynamic podosomelike structure of epithelial cells. Exp Cell Res 295: 360-374, 2004. 
12. Horita Y, Ohashi K, Mukai M, Inoue M and Mizuno K: Suppression of the invasive capacity of rat ascites hepatoma cells by knockdown of Slingshot or LIM kinase. J Biol Chem 283: 6013-6021, 2008

13. Vesely P, Rosel D, Pankova D, Tolde O, Blase C, Matouskova E, Folk P, Brabek J and Bereiter-Hahn J: Confocal microscopy reveals Myzitiras and Vthela morphotypes as new signatures of malignancy progression. Scanning 31: 102-106, 2009.

14. Fabry B, Maksym GN, Shore SA, Moore PE, Panettieri RA Jr, Butler JP and Fredberg JJ: Selected contribution: time course and heterogeneity of contractile responses in cultured human airway smooth muscle cells. J Appl Physiol 91: 986-994, 2001.

15. Raupach C, Zitterbart DP, Mierke CT, Metzner C, Muller FA and Fabry B: Stress fluctuations and motion of cytoskeletal bound markers. Phys Rev E Stat Nonlin Soft Matter Phys 76: 021925, 2007.

16. Mierke CT, Zitterbart DP, Kollmannsberger P, Raupach C, Schlotzer-Schrehardt U, Goecke TW, Behrens J and Fabry B: Breakdown of the endothelial barrier function in tumor cell transmigration. Biophys J 94: 2832-2846, 2008.

17. Chen WT: Proteolytic activity of specialized surface protrusions formed at rosette contact sites of transformed cells. J Exp Zool 251: 167-185, 1989

18. Pankova K, Rosel D, Novotny M and Brabek J: The molecular mechanisms of transition between mesenchymal and amoeboid invasiveness in tumor cells. Cell Mol Life Sci 67: 63-71, 2010.

19. Bursac P, Lenormand G, Fabry B, Oliver M, Weitz DA, Viasnoff V, Butler JP and Fredberg JJ: Cytoskeletal remodelling and slow dynamics in the living cell. Nat Mater 4: 557-561, 2005.

20. Ono S: Mechanism of depolymerization and severing of actin filaments and its significance in cytoskeletal dynamics. Int Rev Cytol 258: 1-82, 2007.

21. Chabottaux V and Noel A: Breast cancer progression: insights into multifaceted matrix metalloproteinases. Clin Exp Metastasis 24: 647-656, 2007.
22. Ulisse S, Baldini E, Sorrenti S and D'Armiento M: The urokinase plasminogen activator system: a target for anticancer therapy. Curr Cancer Drug Targets 9: 32-71, 2009.

23. Sabeh F, Shimizu-Hirota R and Weiss SJ: Protease dependent versus independent cancer cell invasion programs: three dimensional amoeboid movement revisited. J Cell Biol 185: 11-19, 2009.

24. Friedl $\mathrm{P}$ and Wolf $\mathrm{K}$ : Tumour cell invasion and migration: diversity and escape mechanisms. Nat Rev Cancer 3: 362-374, 2003.

25. Mierke CT, Rosel D, Fabry B and Brabek J: Contractile forces in tumor cell migration. Eur J Cell Biol 87: 669-676, 2008.

26. Shiomi T and Okada Y: MT1-MMP and MMP7 in invasion and metastasis of human cancers. Cancer Metastasis Rev 22: 145-152, 2003.

27. Mierke CT, Zitterbart DP, Kollmannsberger P, Raupach C, Schlotzer-Schrehardt U, Goecke TW, Behrens J and Fabry B: Breakdown of the endothelial barrier function in tumor cell transmigration. Biophys J 94: 2832-2846, 2008.

28. Stylli SS, Kaye AH and Lock P: Invadopodia: at the cutting edge of tumour invasion. J Clin Neurosci 15: 725-737, 2008.

29. Condeelis J and Segall JE: Intravital imaging of cell movement in tumours. Nat Rev Cancer 3: 921-930, 2003.

30. Clark ES, Whigham AS, Yarbrough WG and Weaver AM: Cortactin is an essential regulator of matrix metalloproteinase secretion and extracellular matrix degradation in invadopodia. Cancer Res 67: 4227-4235, 2007.

31. Selicharova I, Smutna K, Sanda M, Ubik K, Matouskova E, Bursikova E, Brozova M, Vydra J and Jiracek J: 2-DE analysis of a new human cell line EM-G3 derived from breast cancer progenitor cells and comparison with normal mammary epithelial cells. Proteomics 7: 1549-1559, 2007. 